\title{
GENERATING VARIETIES OF TOPOLOGICAL GROUPS
}

\author{
by M. S. BROOKS, SIDNEY A. MORRIS and STEPHEN A. SAXON
}

(Received 31st December 1971)

\section{Introduction}

Recently several papers on varieties of topological groups have appeared. In this note we investigate the question: if $\Omega$ is a class of topological groups, what topological groups are in the variety $V(\Omega)$ generated by $\Omega$; that is, what topological groups can be " manufactured" from $\Omega$ using repeatedly the operations of taking subgroups, quotient groups and arbitrary cartesian products? We seek a general theorem which will be useful for investigating $V(\Omega)$ for wellknown classes $\Omega$.

Prior to this paper the only result (12) along these lines was the following. If $T$ is the circle group, then the locally compact Hausdorff group $G$ is in $V(T)$ if and only if it is compact and abelian.

Looking to the well-established theory of varieties of groups for " inspiration ", we prove a theorem on generating varieties which yields: If $\Omega$ is a class of compact groups, then any locally compact Hausdorff group in $V(\Omega)$ is compact. Despite this the theorem is of no great interest to us except in that it allows us to prove our main result, Theorem 2 , which is peculiar to the theory of topological varieties. (Analogues for varieties of topological Lie algebras and varieties of linear topological spaces appear in (2) and (4), respectively.)

Our main theorem is crucial to the investigations in (13), (14), (15) and (18). We use it here to answer an open question of (17).

\section{Definitions and results}

If $\Omega$ is a class of (not necessarily Hausdorff) topological groups, then $S$ denotes the class of all topological groups isomorphic to subgroups of members of $\Omega$. Similarly we define the operators $\bar{S}, Q, \bar{Q}, C, C_{0}$ and $D$ where they respectively denote closed subgroup, quotient group, separated quotient group, arbitrary cartesian product, countable product and finite product.

A non-empty class $\Omega$ of topological groups is said to be a variety if $Q \Omega \subset \Omega$, $S \Omega \subset \Omega$ and $C \Omega \subset \Omega$. The smallest variety containing a class $\Omega$ of topological groups is said to be the variety generated by $\Omega$ and is denoted by $V(\Omega)$. (For an excellent treatment of varieties of groups see (19).)

If $V$ is a variety, $X$ is a topological space and $F$ is a member of $V$, then $F$ is said to be a free topological group of $V$ on $X$, denoted by $F(X, V)$, if it has the 
properties:

(a) $X$ is a subspace of $F$,

(b) $X$ generates $F$ algebraically,

(c) for any continuous mapping $\gamma$ of $X$ into a member of $H$ of $V$, there exists a continuous homomorphism $\Gamma$ of $F$ into $H$ such that $\left.\Gamma\right|_{x}=\gamma$.

In (11) it was shown that $F(X, V)$ exists if and only if there is a member of $V$ which has $X$ as a subspace. If $F(X, V)$ exists, then it is unique (up to isomorphism).

Theorem 1. For any non-empty class $\Omega$ of topological groups $V(\Omega)=Q S C \Omega$.

Proof. Firstly we observe the following easily verified statements: $S S \Omega=S \Omega ; \quad C C \Omega=C \Omega ; \quad Q Q \Omega=Q \Omega ; \quad C S \Omega \subset S C \Omega ; \quad C Q \Omega \subset Q C \Omega ;$ $S Q \Omega \subset Q S \Omega$. Using these, we see that $Q S C \Omega$ is a variety by noting that $Q[Q S C \Omega]=Q S C \Omega, S[Q S C \Omega] \subset Q S S C \Omega=Q S C \Omega$ and $C[Q S C \Omega] \subset Q C S C \Omega$ $\subset Q S C C \Omega=Q S C \Omega$.

Note that any variety of groups can be considered as a variety of topological groups simply by endowing each group with its indiscrete topology. Of course, as it applies to varieties of groups the above theorem is well known and our proof is a standard one (see, for example, (7), section 23). However, the usual proof of that case via free groups $((19), 15.23)$ does not extend. The difficulty concerns the following question:

If $\Omega$ is a class of topological groups and $X$ is a Hausdorff space such that $F(X, V(\Omega))$ exists, does $F(X, V(\Omega))$ necessarily belong to $S C \Omega$ ?

The example below, provided by Gerald Edgar and Andrew Gleason, answers this question in the negative.

Example. Let $\Omega$ be the class of all totally disconnected groups and $X$ the real line with its usual topology. Then $S C \Omega=\Omega$ and thus $F(X, V(\Omega)$ ), if it exists, is not in $S C \Omega$.

To see that $F(X, V(\Omega))$ exists, we show that $R \in V(\Omega)$, where $R$ is the additive group of reals with its usual topology (see Corollary 2.10 of (11)). In fact, we show that $R \in Q \Omega$.

In the Banach space $l^{2}$ of real square-summable sequences, let $G$ be the group of sequences which have rational coordinates. Then $G \in \Omega$. Choose any $\alpha \in l^{2}$, with all components non-zero. It is easily seen that the map $f: G \rightarrow R$, given by $f(a)=(a, \alpha)$ where $($,$) is the inner product in l^{2}$, is an open continuous homomorphism of $G$ on to $R$. Thus $R \in Q\{G\} \subset Q \Omega$, as required.

Before presenting our main theorem we state a lemma, the proof of which is omitted as it is straightforward.

Lemma. Let $G$ be a topological group with subgroups $A$ and $B$ such that $B$ is a closed normal subgroup of $A$. Let $\bar{A}$ and $\bar{B}$ be the closures in $G$ of $A$ and $B$ respectively. Then the quotient group $A / B$ is isomorphic to a subgroup of $\bar{A} / \bar{B}$. 
Theorem 2. If $\Omega$ is any class of topological groups and $G$ is a Hausdorff group in $V(\Omega)$ then $G \in S C \bar{Q} \bar{D} D \Omega$.

Proof. We need only show that $G \in S C Q S D \Omega$. (To see this reduction use the lemma together with relevant parts of the proof of Theorem 1 and the easily verified fact that any Hausdorff group in $S C Q \Omega$ is in $S C \bar{Q} \Omega$.)

By Theorem $1, G \in \bar{Q} S C \Omega$. Therefore there exist $K_{i} \in \Omega, i \in I$ such that $K=\pi_{i \in I} K_{i}$ has subgroups $A$ and $B$ with $B$ a closed normal subgroup of $A$ and $G$ isomorphic to the quotient $A / B$. Let $\Sigma$ be the family of all finite subsets of $I$. For $\sigma \in \Sigma$ let $K_{\sigma}=\pi_{i \in \sigma} K_{i}$; let $p_{\sigma}$ be the projection mapping of $K$ onto $K_{\sigma}$; and let $h_{\sigma}$ be the canonical mapping of $p_{\sigma}(A)$ on to the quotient $p_{\sigma}(A) / p_{\sigma}(B)$. Define a map $f: A \rightarrow \pi_{\sigma \in \Sigma} h_{\sigma} p_{\sigma}(A)$ by $f(a)=\pi_{\sigma \in \Sigma}\left(h_{\sigma} p_{\sigma}(a)\right)$ for all $a \in A$; that is, $f$ is the product of $h_{\sigma} p_{\sigma}, \sigma \in \Sigma$, and hence is a continuous homomorphism. For some $\tau \in \Sigma$ let $O=\pi_{i \in I} O_{i}$ where each $O_{i}$ is a neighbourhood of the identity in $K_{i}$ and in fact $O_{i}=K_{i}$ for $i \notin \tau$. We prove $f$ is an open mapping of $A$ onto $f(A)$ by verifying that

$$
f(O \cap A) \supset\left(\pi_{\sigma \in \Sigma} D_{\sigma}\right) \cap f(A)
$$

where $D_{\sigma}=h_{\sigma} p_{\sigma}(A)$ for $\sigma \neq \tau$ and $D_{\tau}=h_{\tau}\left(p_{\tau}(O) \cap p_{\tau}(A)\right)$. If

$$
x \in\left(\pi_{\sigma \in \Sigma} D_{\sigma}\right) \cap f(A)
$$

then there exists $a \in A$ such that $x=f(a) \in \pi_{\sigma \in \Sigma} D_{\sigma}$, and so there exists $c \in O$ such that $h_{\tau} p_{\tau}(c)=h_{\tau} p_{\tau}(a)$; that is, $p_{\tau}(B) p_{\tau}(c)=p_{\tau}(B) p_{\tau}(a)$. So there exists $b \in B$ such that $p_{\tau}(c)=p_{\tau}(b) p_{\tau}(a)=p_{\tau}(b a)$. But this means that $p_{\tau}(b a) \in p_{\tau}(O)$ and therefore that $b a \in O$. This shows that $x \in f(O \cap A)$ since clearly $B$ is in the kernel of $f$ and so $x=f(a)=f(b a)$.

We now show that the kernel of $f$ is in $B$. If $y \notin B$ then, since $B$ is closed in $A$, there exists a neighbourhood $N=\pi_{i \in I} N_{i}$ of $y$ in $\pi_{i \in I} K_{i}$ with $N \cap B=\varnothing$ and $N_{i}=K_{i}$ for all $i \notin \gamma$, where $\gamma$ is suitably chosen from $\Sigma$.

Clearly $p_{y}(N) \cap p_{\gamma}(B)=\varnothing$, and since $p_{\gamma}(y) \in p_{\gamma}(N)$ it follows that $p_{\gamma}(y) \notin p_{\gamma}(B)$ Since $p_{y}(B)$ is the kernel of $h_{y}$, this shows that $f(y)=\left(h_{a} p_{a}(y)\right)_{\sigma \in \Sigma}$ is not the identity.

Thus we have that $f$ is an open continuous homomorphism with kernel $B$, and so $A / B$ is isomorphic to a subgroup of $\pi_{\sigma \in \Sigma} h_{\sigma} p_{\sigma}(A)$; that is, $G \in S C Q S D \Omega$, as we set out to show.

Remark. In the abelian case the commutativity of the operators $Q$ and $S$ yields: If $\Omega$ is any class of abelian topological groups and $G$ is a Hausdorff group in $V(\Omega)$ then $G \in S C \bar{Q} D \Omega$. We do not know whether the "abelian" condition is necessary or not.

Corollary 1. If $\Omega$ is a class of locally compact Hausdorff groups or a class of complete metrizable groups then every Hausdorff group $G$ in $V(\Omega)$ has a completion $\hat{G}$ and $\hat{G}$ is in $V(\Omega)$.

Proof. If $\Omega$ consists either of locally compact Hausdorff groups or of complete metrizable groups then $\bar{Q} \bar{S} D \Omega$ contains only groups of the same kind. 
Since the product of complete groups is complete it now follows from Theorem 2 that $G$ is a subgroup of a complete group in $V(\Omega)$.

Remarks. We note that not every variety is closed under the formation of completions-for example, the variety generated by the additive group of rationals with its usual topology.

It is known ((1), Exercise 16c) that not every topological group has a completion. It therefore follows that the variety of all topological groups is not generated by the class of all locally compact Hausdorff groups. Our next corollary shows that the variety of all abelian topological groups is not generated by its locally compact Hausdorff groups. (Further information is obtained in (16).)

Corollary 2. If $\Omega$ is any class of locally compact abelian groups, then any Hausdorff group in $V(\Omega)$ is maximally almost periodic.

Proof. We abbreviate maximally almost periodic to MAP. By Theorem 2, $G \in S C \bar{Q} \bar{S} D \Omega$. Now $\bar{Q} \bar{S} D \Omega$ contains only locally compact Hausdorff abelian groups and these are MAP. To see that $G$ is MAP we only have to note that products and subgroups of MAP groups are MAP.

Remark. It is noted in (18) that a variety generated by locally compact MAP groups can contain (locally compact) Hausdorff non-MAP groups.

Our next corollary could have been derived easily from Theorem 1.

Corollary 3. If $\Omega$ is any class of pseudocompact Hausdorff groups, then every complete Hausdorff (in particular, locally compact Hausdorff) group $G$ in $V(\Omega)$ is compact.

Proof. It is shown in (3) and (20) that every member of $\Omega$ can be embedded in a compact Hausdorff group. Thus $V(\Omega) \subset V(\Gamma)$, where $\Gamma$ is the class of all compact Hausdorff groups. Since $G$ is complete, Theorem 2 implies that $G \in \bar{S} C \bar{Q} \bar{S} D \Gamma=\Gamma$.

Remark. It was shown in (6) that if $X$ is compact Hausdorff and $V$ is the variety of all (all abelian) topological groups, then $F(X, V)$ exists and is complete Hausdorff. Corollary 3 shows that if $\Omega$ is any class of connected compact Hausdorff groups then $F(X, V(\Omega)$ ) is not complete. (It is shown in (17) that $F(X, V(\Omega))$ exists and is Hausdorff. Therefore by Corollary 3 , if it is complete then it is compact. However, by Theorem 2.8 of (11) and Theorem 2.5 (iv) of (2) $F(X, V(\Omega))$ is algebraically a free group or a free abelian group and consequently, (5), does not admit a compact Hausdorff topology.)

Corollary 4. If $\Omega$ is a class of locally compact Hausdorff groups, then the following conditions are equivalent:

(i) $R \in S \Omega$

(ii) $R \in V(\Omega)$

(iii) $V(\Omega)$ contains a connected non-compact locally compact Hausdorff group. 
Proof. Clearly (i) implies (ii) and (ii) implies (iii). Now suppose (iii) is true and (i) is false. Then there exists a connected non-compact locally compact Hausdorff group $G$ in $V(\Omega)$. According to $\S 4.13$ of $(\mathbf{1 0}), G$ has $R$ as a subgroup and so $R \in V(\Omega)$.

Since $R \notin S \Omega$, the component of the identity of each member of $\Omega$ is compact (again using $\$ 4.13$ of (10)). Clearly then this is also true for each member of $\bar{S} D \Omega$. Indeed by 7.12 of (9) it is true for each member of $\bar{Q} \bar{S} D \Omega$.

By Theorem 2, $R \in V(\Omega)$ implies $R \in S C \bar{Q} \bar{S} D \Omega$; that is, $R$ is a subgroup of a product $\pi_{i \in I} G_{i}$, where each $G_{i} \in \bar{Q} \bar{S} D \Omega$ and hence has compact component $K_{i}$ of the identity. Since $R$ is connected, its projection into $G_{i}$ is contained in $K_{i}$. Hence $R$ is a subgroup of the compact group $\pi_{i \in I} C_{i}$, which is impossible. Thus (iii) implies (i).

Remarks. It was proved in (12) that $R \notin V(T), R \notin V(Z)$ and $Z \notin V(T)$, where $T$ and $Z$ respectively denote the circle group and the group of integers with the usual topology. We now see from Corollary 4 that $R \notin V\{Z, T\}$. Indeed, by Corollary 1, the additive group of rationals with its usual topology is not in $V\{Z, T\}$.

We can use this to answer an "open question" in (17). First we need a definition: A variety $V$ is said to be a $\beta$-variety if for every compact Hausdorff space $X, F(X, V)$ exists and is Hausdorff. It is shown in (17) that any variety generated by arcwise connected Hausdorff groups is a $\beta$-variety. The "open question " asks if the converse is true. We answer this in the negative by proving that the $\beta$-variety $V\{Z, T\}$ is not generated by its connected Hausdorff groups. Indeed the corollary below says somewhat more.

Corollary 5. Let $\Omega$ be a class of locally compact abelian groups. Then $V(\Omega)$ is generated by its connected Hausdorff groups if and only if $V(\Omega)$ equals $V(R)$ or $V(T)$.

Proof. If $G$ is any connected Hausdorff group in $V(\Omega)$, then $G \in S C \bar{Q} \bar{S} D \Omega$; that is, $G \in S C\left\{H_{\alpha}: \alpha \in I\right\}$, where each $H_{\alpha} \in \bar{S} \bar{Q} \bar{S} D \Omega$ and the projection of $G$ is dense in $H_{a}$. Thus $H_{a}$ is connected and locally compact. Hence $V(\Omega)$ is generated by its connected locally compact Hausdorff groups. Since every such group is a projective limit of Lie groups, we have that $V(\Omega)$ is generated by connected abelian Lie groups. These groups are of the form $R^{a} \times T^{b}$, where $a$ and $b$ are non-negative integers. So $V(\Omega)$ equals $V(R)$ or $V(T)$, as required.

Remark. Let $\Omega$ be a class of locally compact groups. Then, as above, $V(\Omega)$ is generated by its connected Hausdorff groups if and only if it is generated by its connected Lie groups. Corollary 7 then says that every discrete group in $V(\Omega)$ is countable. Thus if $\Omega$ is the class of all locally compact groups, $V(\Omega)$ is not generated by its connected Hausdorff groups. So we have another proof that $V(\Omega)$ is a proper subvariety of the variety of all topological groups as it was shown in (8) that the latter variety is generated by connected Hausdorff groups. 
Corollary 6. If $\Omega$ is any class of topological groups and $G$ is a metrizable group in $V(\Omega)$ then $G \in S C_{0} \bar{Q} \bar{S} D \Omega$.

Proof. By Theorem 2 we need only show that if a metrizable group $G$ is a subgroup of a product $\pi_{i \in I} H_{i}$ of topological groups $H_{i}$, then there exists a countable subset $J$ of $I$ such that $G$ is isomorphic to a subgroup of $\pi_{i \in J} H_{i}$.

Now $G$ has a base of neighbourhoods $N_{j}, j=1,2, \ldots$ of the identity element such that $N_{j}=\left(\pi_{i \in I} D_{i j}\right) \cap G$, where each $D_{i j}$ is a neighbourhood of the identity in $H_{i}$ and there is a finite subset $I_{j}$ of $I$ with $D_{i j}=H_{i}$ for all $i \notin I_{j}$. Put $J=\bigcup_{j=1}^{\infty} I_{j}$ and let $f$ be the natural projection of $\pi_{i \in I} H_{i}$ on to $\pi_{i \in J} H_{l}$. It is now a routine matter to verify that $f(G)$ is isomorphic to $G$.

As an immediate consequence we have.

Corollary 7. If $\Omega$ is a class of separable metric groups and $G$ is a metrizable group in $V(\Omega)$ then $G$ is separable.

\section{Acknowledgement}

The authors thank Joe Diestel and Aaron Todd for their stimulating comments.

\section{REFERENCES}

(1) N. BourbakI, General topology (Addison-Wesley Co., Reading, Massachusetts, 1966).

(2) Su-shing Chen and Sidney A. Morris, Varieties of topological groups generated by Lie groups, Proc. Edinburgh Math. Soc. (2) 18 (1972), 49-53.

(3) W. W. Comfort and K. A. Ross, Pseudocompactness and uniform continuity and topological groups, Pacific J. Math. 16 (1966), 483-496.

(4) J. Diestel, Stephen A. SAXon and Sidney A. Morris, Varieties of linear topological spaces, Trans. Amer. Math. Soc. 171 (1972) (to appear).

(5) R. M. Dudley, Continuity of homomorphism, Duke Math. J. 28 (1961), 587-594.

(6) M. I. Graev, Free topological groups, Izvestiya Akad. Nauk SSR. Ser. Mat. 12 (1948), 279-324, (Russian): Amer. Math. Soc. Translation No. 35, (1951), reprinted in Amer. Math. Soc. Transl. (1) 8 (1962), 305-364.

(7) G. GRÄTzER, Universal Algebra (Van Nostrand, 1968).

(8) S. HARTMAN and JAN MYcIELSKI, On the imbedding of topological groups into connected topological groups, Coll. Math. 5 (1958), 167-169.

(9) E. HewITT and K. A. Ross, Abstract harmonic analysis I (Academic Press, New York, 1963).

(10) D. MONTGOMERY and L. ZIPPN, Topological transformation groups (Interscience, New York, 1955). 
(11) Sidney A. Morris, Varieties of topological groups, Bull. Austral. Math. Soc. 1 (1969), 145-160.

(12) Sidney A. Morris, Varieties of topological groups HI, Bull. Austral. Math. Soc. 2 (1970), 165-178.

(13) Sidney A. MorRIs, Locally compact abelian groups and the variety of topological groups generated by the reals, Proc. Amer. Math. Soc. 34 (1972), 290-292.

(14) SIDNEy A. Morris, On varieties of topological groups generated by solvable groups, Coll. Math. (1972).

(15) SIDNEY A. Morris, Varieties of topological groups generated by solvable and nilpotent groups, Coll. Math. (to appear).

(16) Sidney A. Morris, A topological group characterization of those locally convex spaces having their weak topology, Math. Ann. 195 (1972), 330-331.

(17) Sidney A. MoRris, Locally compact groups and $\beta$-varieties of topological groups, Fundamenta Mathematicae (to appear).

(18) SIDNEY A. MoRris, Varieties of topological groups generated by maximally almost periodic groups, Fundamenta Mathematicae (to appear).

(19) Hanna Neumann, Varieties of groups (Ergebnisse der Mathematik und ihrer Grenzgebiete, Band 37, Springer-Verlag, Berlin-Heidelberg, New York, 1967).

(20) Rodney Nillsen, Compactification of products, Mat. Casopis Sloven. Akad. Vied, 19 (1969), 316-323.

Canberra College of Advanced Education

Canberra, A.C.T., 2600

Australia

UNIVERSITY OF NEW SOUTH WALES

Kensington, N.S.W., 2033

Australia

AND

UNIVERSITY OF FLORIDA

Gainesville, Florida, 32601

U.S.A. 